\title{
New secure and low-cost design for defense in depth implementation using open source software
}

\begin{abstract}
In this paper, we propose a new design with high-level security and low-cost implementation for different network topologies. Achieving both factors at the same time is a challenging work for network experts, especially when they want to apply defense in depth strategy in various components of their networks. Offered scenario has been done for one of the most visited web sites in an Iranian organization with more than 15,000 concurrent web users that all of them reliably and smoothly were served. Also, defense in depth strategy has been used to provide sufficient level of security in diverse components of the project. All applied security solutions in this project were based on open source [1] tools that results in saving a tangible cost by the prevention of purchasing commercial options. We will describe project big map, acquired results and all main components with their functions in this paper.
\end{abstract}

Keyword: Cisco; DMZ; Firewall; IDS; Linux; Open source 